\title{
Avoidance of Maxillary Swing for Nasopharyngectomy via Combined Open Lateral and Endoscopic Approach
}

\author{
S. Hamad Sagheer, BS \\ Thomas Jefferson University \\ Brian Swendseid, MD \\ Thomas Jefferson University \\ Joseph M. Curry, MD \\ Thomas Jefferson University \\ Mindy Rabinowitz, MD \\ Thomas Jefferson University \\ Gurston Nyquist, MD

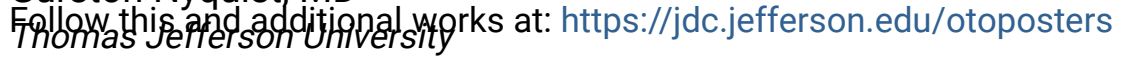 \\ Part of the Otolaryngology Commons \\ bet us. knowhingw access to this document benefits you
}

\section{Recommended Citation}

Sagheer, BS, S. Hamad; Swendseid, MD, Brian; Curry, MD, Joseph M.; Rabinowitz, MD, Mindy; Nyquist, MD, Gurston; Rosen, MD, Marc R.; Evans, MD, James; and Luginbuhl, Adam J., "Avoidance of Maxillary Swing for Nasopharyngectomy via Combined Open Lateral and Endoscopic Approach" (2021). Department of Otolaryngology - Head and Neck Surgery Posters. 10.

https://jdc.jefferson.edu/otoposters/10

This Article is brought to you for free and open access by the Jefferson Digital Commons. The Jefferson Digital Commons is a service of Thomas Jefferson University's Center for Teaching and Learning (CTL). The Commons is a showcase for Jefferson books and journals, peer-reviewed scholarly publications, unique historical collections from the University archives, and teaching tools. The Jefferson Digital Commons allows researchers and interested readers anywhere in the world to learn about and keep up to date with Jefferson scholarship. This article has been accepted for inclusion in Department of Otolaryngology - Head and Neck Surgery Posters by an authorized administrator of the Jefferson Digital Commons. For more information, please contact: JeffersonDigitalCommons@jefferson.edu. 


\section{Authors}

S. Hamad Sagheer, BS; Brian Swendseid, MD; Joseph M. Curry, MD; Mindy Rabinowitz, MD; Gurston Nyquist, MD; Marc R. Rosen, MD; James Evans, MD; and Adam J. Luginbuhl 


\title{
Avoidance of Maxillary Swing for Nasopharyngectomy via \\ Combined Open Lateral and Endoscopic Approach
}

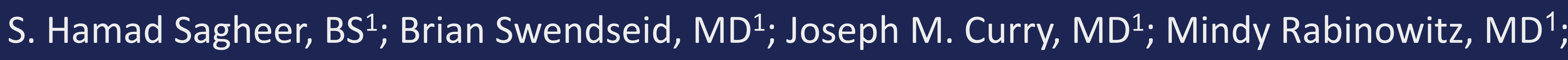 \\ Gurston Nyquist, MD1; Marc R. Rosen, MD ; James Evans, MD ${ }^{1,2}$; Adam J. Luginbuhl, MD ${ }^{1}$ \\ ${ }^{1}$ Department of Otolaryngology - Head and Neck Surgery, Thomas Jefferson University Hospital; \\ ${ }^{2}$ Department of Neurological Surgery, Thomas Jefferson University Hospital
}

\begin{abstract}
Objectives: Nasopharyngectomy performed via a solely endoscopic approach has limitations in access and feasibility, particularly regarding management of the carotid artery. To address these limitations, we report three cases with one cadaver dissection where nasopharyngectomy was performed via a combined open lateral an endoscopic approach. We highlight the benefits and technical considerations for this operative technique. Study Design: Case Series

Methods: Patients diagnosed with recurrent nasopharyngeal carcinoma (NPC) that underwent combined open lateral and endoscopic nasopharyngectomy from 2016-2020 were analyzed. A cadaver dissection was also performed.

Results: We present the details of the approach and follow-up in three patients

with recurrent nasopharyngeal carcinoma. Briefly, a preauricular incision is extended down to the neck. The zygoma and mandibular ramus can be removed and replaced if required. V3, the pterygoid plates and the eustachian tube can be resected or mobilized. The carotid artery may be identified proximally in the neck and traced to the skull base, where the carotid canal may be drilled to the level of the foramen lacerum and protected with pledget. Then, tumor mucosal cuts are made via an endoscopic endonasal approach and connected to the lateral exposure. No carotid artery injuries occurred.

Conclusion: The combined open lateral approach and endoscopic nasopharyngectomy technique is a useful technique in salvage patients. It provides excellent control of majo vessels, adequate access to the carotid canal, V3, and remainder of the skull base, and cervical protection
\end{abstract}

\section{Introduction}

- Endoscopic approaches to the nasopharynx are often performed when feasible due to lower patient morbidity. ${ }^{1}$

- More extensive or recurrent tumors may require open approaches, with a maxillary swing representing the most common option. Maxillary swing provides access to the nasopharynx and ipsilateral parapharyngeal space $^{2}$, with limited access to the skull base and exposure of the internal carotid artery.

- There is added morbidity owing to the transfacial incision, including skin, infraorbital nerve, and palate resection. ${ }^{3}$

- Combined open lateral and endoscopic approach to the skull base, may avoid morbidity and improve exposure in select patients.

\section{Methods}

- Retrospective review was performed to identify patients with a nasopharyngeal carcinoma (NPC) that then underwent combined open lateral approach and endoscopic transnasal nasopharyngectomy.

- Patients with purely endoscopic approaches were excluded.

- Patients were identified from 2016-2020, and their electronic medical records were reviewed to characterize their post-operative course.

\section{Results}

- The open lateral approach consists of a preauricular incision extending down to the neck.

- The facial nerve is kept within the parotid and mobilized superiorly to decrease traction.

- The zygoma and mandibular ramus can be removed and replaced if required. With reflection of the facial nerve inferiorly within the parotid, allowing a corridor along the infratemporal fossa.

- This exposure allows dissection to proceed along the skull base, where V3, the pterygoid plates and the eustachian tube can be resected or mobilized.

- The carotid artery may be identified proximally in the neck and traced to the skull base. The carotid canal may be drilled to the level of the foramen lacerum through the superior corridor after removal of the zygoma. A pledget is placed over the medial carotid for protection.

- Then, tumor mucosal cuts are made via an endoscopic endonasal approach, which are carried to the open infratemporal fossa dissection.

- Once the two approaches are connected, further drilling of the carotid canal can be achieved via either approach. No carotid artery injuries occurred.

- With a mean follow-up of 7.9 (range $2.6-15.4$ ) months, no evidence of cerebrospinal fluid (CSF) leak, meningitis, carotid injury, or tumor recurrence at the site of reconstruction was reported.
Figure 1. Cadaveric dissection of the left preauricular approach revealing the carotid artery $(*)$ and carotid canal $\left({ }^{* *}\right)$.
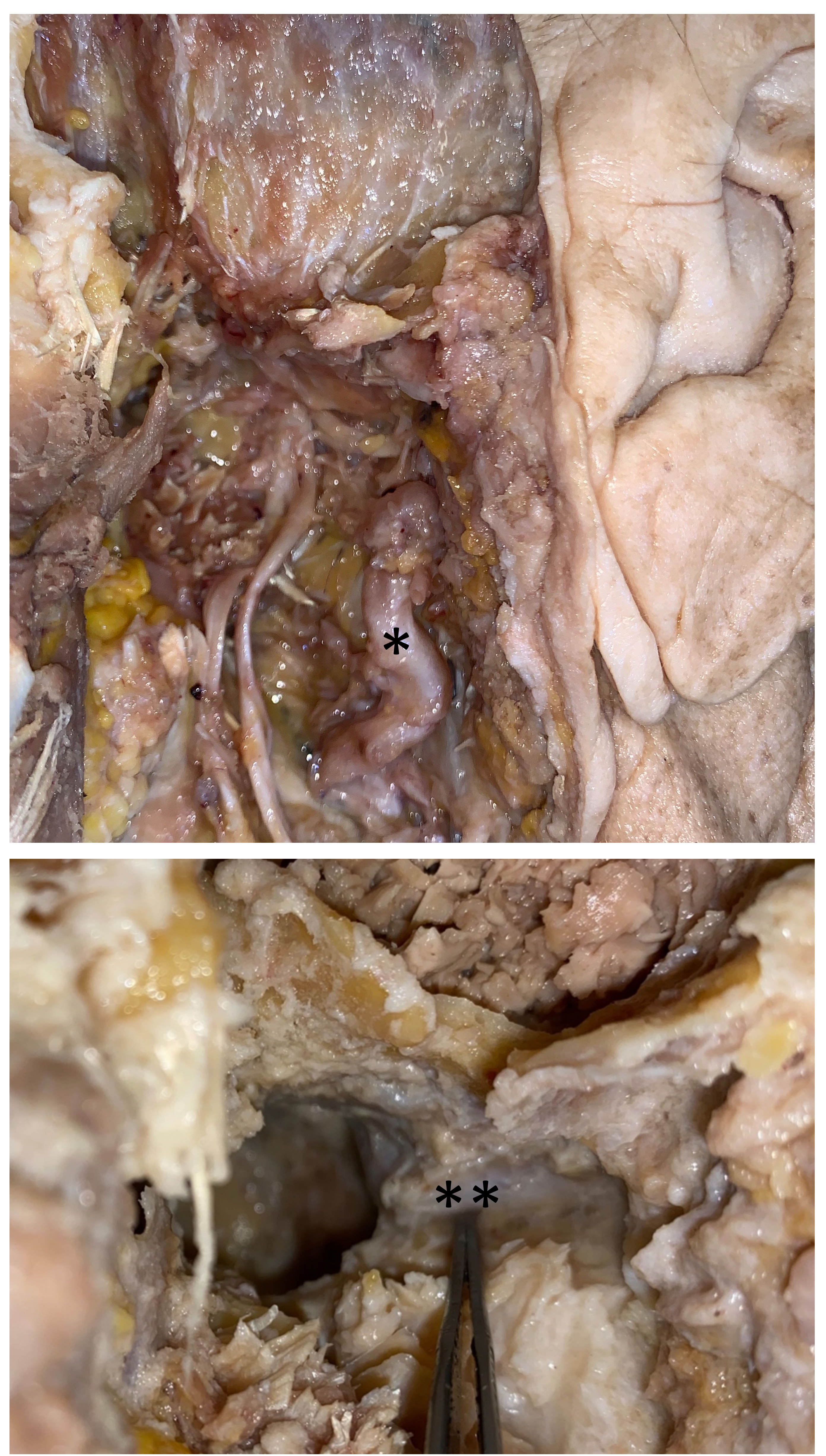

\section{Discussion}

- Management of the carotid artery through the carotid canal can be achieved via the transcervical approach, allowing for confident tumor dissection endoscopically.

- Extended drilling of the canal to the foramen lacerum is accomplished through either approach, but pterygoid plates are resected through the lateral approach.

- The Eustachian tube isthmus and pterygoid plate are known surgical landmarks of the carotid artery and can be identified via open latera approach. ${ }^{4}$

- Short-term outcomes following this surgical technique are encouraging but long-term surveillance in a larger patient cohort is required.

\section{Conclusions}

- The combined endoscopic and open lateral approach nasopharyngectomy technique without a maxillary swing is a useful technique in previously operated and irradiated patients with recurrent nasopharyngeal cancer.

- This technique avoids the morbidity of transfacial access for maxillary swing, with likely improved carotid artery control and skull base visualization.

\section{Contact}

\section{References}

Adam Luginbuhl, MD

Thomas Jefferson University Hospital

925 Chestnut St.

adam.luginbuhl@jefferson.edu

215 955-5208

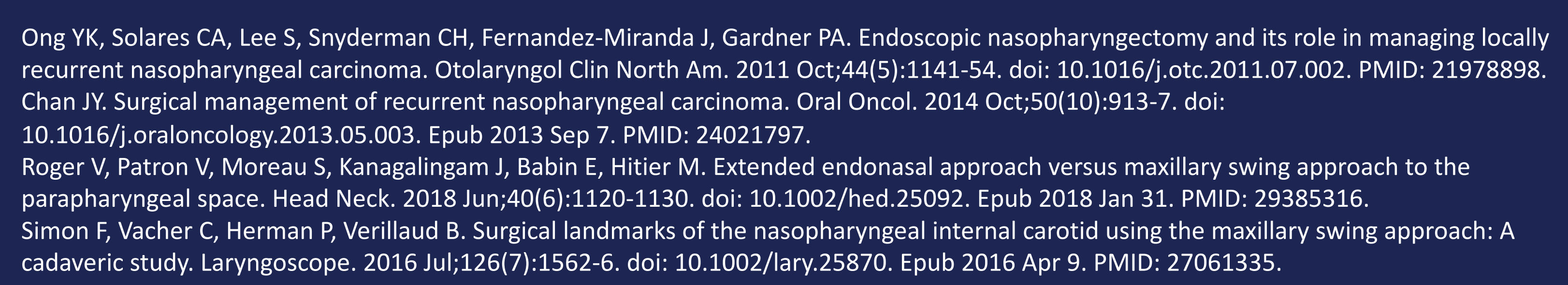

